\title{
Cerebral fat embolism after traumatic bone fractures: a structured literature review and analysis of published case reports
}

Luigi Vetrugno ${ }^{1,2^{*}}$ (D) Elena Bignami ${ }^{3}$, Cristian Deana $^{4}$, Flavio Bassi ${ }^{5}$, Maria Vargas ${ }^{6}$, Maria Orsaria ${ }^{7}$, Daniele Bagatto ${ }^{8}$, Cristina Intermite ${ }^{2}$, Francesco Meroi ${ }^{2}$, Francesco Saglietti ${ }^{9}$, Marco Sartori ${ }^{2}$, Daniele Orso ${ }^{2}$, Massimo Robiony ${ }^{10,11}$ and Tiziana Bove $e^{1,2}$

\begin{abstract}
Background: The incidence of cerebral fat embolism (CFE) ranges from $0.9-11 \%$, with a mean mortality rate of around $10 \%$. Although no univocal explanation has been identified for the resulting fat embolism syndrome (FES), two hypotheses are widely thought: the 'mechanical theory', and the 'chemical theory'. The present article provides a systematic review of published case reports of FES following a bone fracture.

Methods: We searched MEDLINE, Web of Science and Scopus to find any article related to FES. Inclusion criteria were: trauma patients; age $\geq 18$ years; and the clinical diagnosis of CFE or FES. Studies were excluded if the bone fracture site was not specified.

Results: One hundred and seventy studies were included (268 cases). The male gender was most prominent $(81.6 \%$ vs. $18.4 \%)$. The average age was 33 years $( \pm 18)$. The mean age for males $(29 \pm 14)$ was significantly lower than for females $(51 \pm 26)(p<0.001)$. The femur was the most common fracture site ( $71 \%$ of cases). PFO was found in $12 \%$ of all cases. Univariate and multivariate regression analyses showed the male gender to be a risk factor for FES: RR 1.87 and 1.41, respectively (95\%Cl 1.27-2.48, $p<0.001 ; 95 \% \mathrm{Cl} 0.48-2.34, p<0.001)$.

Conclusions: FES is most frequent in young men in the third decades of life following multiple leg fractures. FES may be more frequent after a burst fracture. The presence of PFO may be responsible for the acute presentation of cerebral embolisms, whereas FES is mostly delayed by $48-72 \mathrm{~h}$.
\end{abstract}

Keywords: Cerebral fat embolism, Fat embolism syndrome, Patent foramen ovale

\section{Introduction}

Cerebral fat embolism (CFE) is a rare and potentially fatal condition that may occur following a long bone fracture or pelvis trauma, showing an incidence ranging from 0.9 to $11 \%$ with a mean mortality rate around $10 \%$

\footnotetext{
* Correspondence: luigi.vetrugno@uniud.it

${ }^{1}$ Department of Anesthesia and Intensive Care, Anesthesia and Intensive Care Clinic, Azienda Sanitaria Universitaria Friuli Centrale, Udine, Italy

${ }^{2}$ Department of Medicine, Anesthesia and Intensive Care Clinic, University of Udine, Udine, Italy

Full list of author information is available at the end of the article
}

[1-3]. After a 'bone burst', according to the accepted pathophysiology, 'fat droplets' are shot into the systemic circulation, giving rise to emboli $[4,5]$. But no univocal explanation exists to describe how the syndrome develops from hereon. Two hypotheses have been validated to account for the early and late symptoms, respectively. The "mechanical theory" describes marrow fat being pushed into the veins as a result of the increased intramedullary pressure, generating respiratory and neurological changes and the emergence of non-palpable skin petechiae in the upper body area (axillae, trunk and

(c) The Author(s). 2021 Open Access This article is licensed under a Creative Commons Attribution 4.0 International License, which permits use, sharing, adaptation, distribution and reproduction in any medium or format, as long as you give appropriate credit to the original author(s) and the source, provide a link to the Creative Commons licence, and indicate if changes were made. The images or other third party material in this article are included in the article's Creative Commons licence, unless indicated otherwise in a credit line to the material. If material is not included in the article's Creative Commons licence and your intended use is not permitted by statutory regulation or exceeds the permitted use, you will need to obtain permission directly from the copyright holder. To view a copy of this licence, visit http://creativecommons.org/licenses/by/4.0/. The Creative Commons Public Domain Dedication waiver (http://creativecommons.org/publicdomain/zero/1.0/) applies to the data made available in this article, unless otherwise stated in a credit line to the data. 
sclera) 2 or 3 days following the trauma [6, 7]. This triad of symptoms is known as fat embolism syndrome (FES). The most common sub-clinical presentation of FES can be reasonably identified as a reduction in respiratory gasexchange and petechiae $[8,9]$, whereas the most severe "mechanical" scenario results in acute respiratory symptoms, acute right heart failure (Peltier's theory) [10] and even abrupt brain death. The "chemical theory" is built on evidence showing that products of the systemic inflammatory response, secondary to the initial bone trauma, trigger the conversion of fat molecules into free fatty acids and glycerol that can, in turn, lead to vascular lesions [11, 12]. This chemical theory is able to account for the vascular endothelial symptoms of the affected region and explains the late signs of central nervous system damage: confusion, hemiplegia, apraxia, aphasia and lethargy. The characteristic "starry night" magnetic resonance image (MRI) of the brain appears approx. One week after the trauma [13]. In the first case, the most accredited events are: highvelocity trauma; the deferral of surgery for more than 10$24 \mathrm{~h}$; multiple fractures; and, as co-factor, difficulties in fracture reduction and nailing or external fixation [14]. The potential correlation between the presence of patent foramen ovale (PFO) and FES in the latter case has yet to be explored $[15,16]$. In the absence of a right-to-left shunt, fat globules would have to be small and multiple to generate a neurological presentation [17]. On the other hand, once tiny fat particles have traversed the pulmonary capillary bed, they can then reach the brain. To the best of our knowledge, the significance of a PFO has never been formally reviewed in the context of FES. Nevertheless, we suggest likening the force and result of the trauma to a fairground "test your strength" hammer game (Fig. 1). The more 'favorable' mechanical scenario depends on the low energy of the fracture-causing 'strike' in which the resulting fat embolism is not 'strong enough' to trigger cerebral fat embolism CFE or FES. By contrast, a more 'critical' mechanical scenario would be one in which a high energy blow results in the fat being 'shot' as far as the pulmonary capillary bed or central nervous system.

The recent literature highlights how FES has gathered recognition thanks to the clinical descriptions in case reports and case series [18-22]. However, the frequency of the syndrome is too low to permit any conclusions to be drawn from the individual studies reported in literature. Adopting a structured literature review approach, we analyzed all the relevant studies available in the literature. We aimed to provide a review of CFE occurring after a bone fracture, combined with separate examinations of published cases in adult patients. Specifically, we focused attention on identifying the associated risk factors, the laterality of the fractures, the presence of PFO, any cerebral involvement and the pathophysiology of CFE.

\section{Materials and methods}

\section{Article selection}

We conducted a literature analysis between 8th October 2018 and 8th December 2018, applying the following

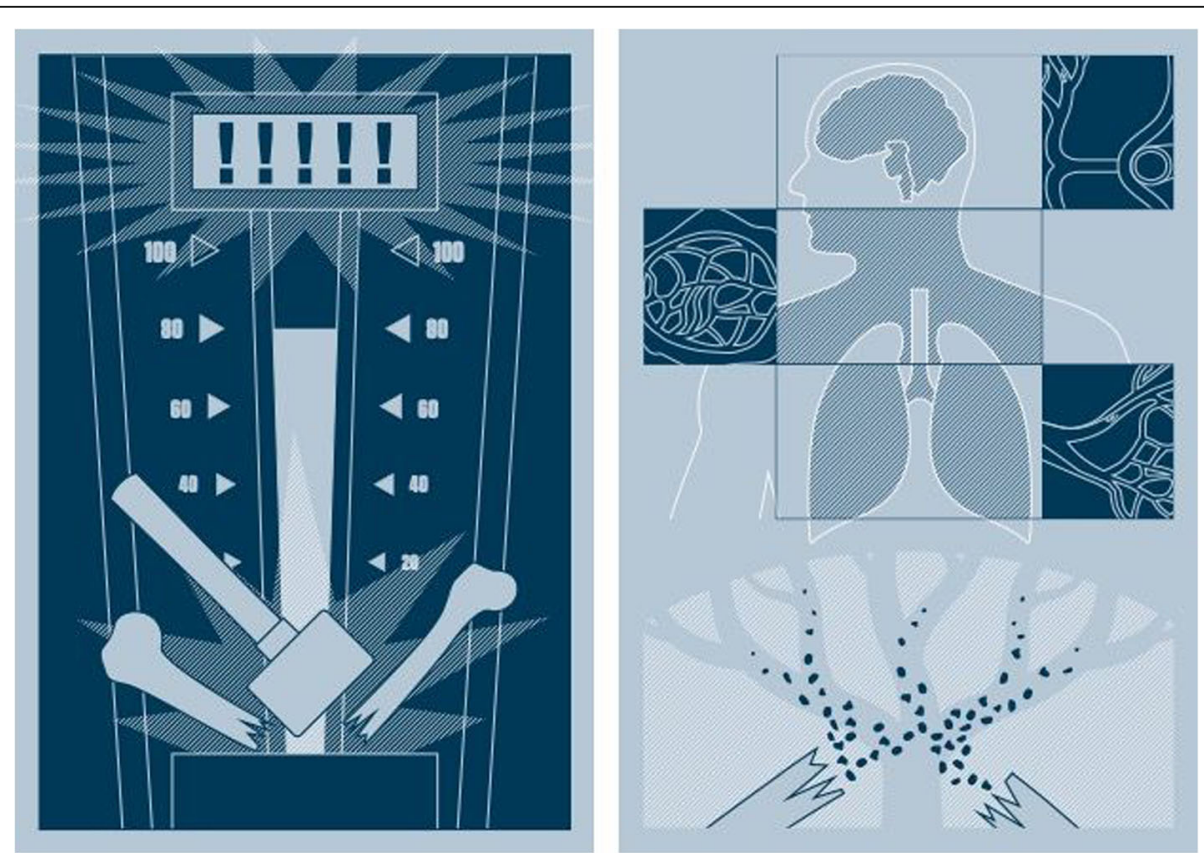

Fig. 1 A simple model based on a fairground 'test your strength' hammer game might explain why higher energy impacts are more likely to result in fat emboli being 'shot' into the vascular compartment able to reach the upper body. Multiple organ involvement of fat embolization according to Peltier's mechanical theory 
search terms: "cerebral fat embolism AND fat embolism syndrome", "cerebral fat embolism OR fat embolism syndrome", "cerebral fat embolism OR fat embolism syndrome AND case report" and "cerebral fat embolism AND fat embolism syndrome AND case report" to search the MEDLINE (1960-2018), SCOPUS (19602018) and WEB OF SCIENCES (1960-2018) databases. Peer-reviewed studies written in English and presumed to report human cases of CFE in adult patients were selected based upon the Preferred Reporting Items for Systematic Review and Meta-Analyses (PRISMA) protocol [23]. This systematic review was formerly registered in the prospective international register of systematic reviews - PROSPERO - date: 30th January 2019 - with the identification number \#CRD42019114334.

\section{Data collecting and handling}

All articles were selected, screened and reviewed, and the data from each case report collated and registered into a Microsoft Excel (Microsoft, Redmond, Washington, USA) spreadsheet. The data included: age; sex; body mass index (BMI); the site, number and side (right or left or bilateral) of bone fracture; the presence of PFO; and the location and side of cerebral involvement diagnosed with computed tomography (CT) or a magnetic resonance image (MRI) brain scan. In the case that some features were absent, data were coded as 'missing'.

\section{Study selection and eligibility criteria}

Inclusion criteria were: trauma patients; age $\geq 18$ years; and clinical diagnosis of CFE or FES. Studies were excluded in the absence of data about the bone fracture site. Five authors (C.I., F. M, F. S, C.D. and M.S.) carried out two independent searches to identify eligible studies for the pooled analysis. The data retrieved by the researchers from these studies were compared. The eligibility of each study was decided by all five researchers. We established a priori that all disputes would be resolved through the consultation of the senior investigator (L.V.).

\section{Statistical analysis}

The following data were collected from each study: sex; age; presence of PFO patency; presence of a brain CT scan; presence of a brain nuclear magnetic resonance image; localization of brain lesions; and localization of various fractures. Data were reported as means \pm standard deviations (S.D.), medians and interquartile ranges, or proportions as appropriate. Comparisons between groups were performed using the Mann-Whitney U test or two-way ANOVA for continuous variables, and Fisher's test or the $\chi^{2}$ test for categorical variables. For the analysis, we divided patients according to sex and age. Univariate logistic regression was performed to identify clinically essential variables potentially associated with CFE between males and females and between different ages. Multivariate logistic regressions were carried out using backward stepwise variable elimination and included the variables that reached a statistical significance at the univariate logistic regression. Statistical significance was set at $\alpha=0.05$. Statistical analysis was performed using SPSS (version 20.0, IBM ${ }^{\circ}$ ).

\section{Results}

We identified a total of 170 research studies for review as depicted in the PRISMA flow diagram (Figure S1 on Supplementary Material), providing a total of 268 cases of FES (Table S1 on Supplementary Material). As expected, gender and age were dominant risk factors, with males more at risk than females $(81.6 \%$ vs. $18.4 \%)$. The average age of all patients was 33 years $( \pm 18)$, whereas the mean age of males was significantly lower than that for females, at $29( \pm 14)$ versus $51( \pm 26)$ years, respectively $(p<0.001)$, despite a spectrum of diverse attitude and frequency in the two classes (Table S2 on Supplementary Material). In particular, the age category containing the highest proportion of male cases was 21-30 years, whereas female cases were more equally distributed between age categories, although the highest proportion fell into the $71-80$ age range. In the preliminary analysis there were a very small number of traumatic pediatric cases: 8 cases in total aged $<18$ years $(1$ case 13 years, 1 case 15 years, 1 case 16 years, 5 cases 17 years). A bar chart demonstrating the distribution for each gender according to age is presented in Figure S2 (supplementary material) .

Regarding to fracture site, $71 \%$ of cases involved the femur, $36 \%$ the tibia, and $19 \%$ the fibula (isolated or crus fracture). Other fracture sites (e.g. radius, ulna, ribs) exhibited incidences of less than 10\% (Table S3 on Supplementary Material). The fractures were more often bilateral in males, with higher involvement of the left side compared with females (21.4 vs $4.2 ; p=0.031$ ). However, the right side was the most frequently involved in both sexes (Table S4 on Supplementary Material). Examining the fracture sites according to age category, lower limb fractures constitute the most common fractures in all age groups, whereas fractures of the upper limbs were most common in the between 19 to 30 age group. Fractures of the chest wall were most prevailing between 51 and 60 years (Table S5 on Supplementary Material). Concerning the presentation of FES as confirmed by CT or MR imaging (Table S6 on Supplementary Material), the most involved sites were the internal capsule (82.4\%) and subcortical zones (89\%). The most affected brain lobes were the frontal (31.7\%) and parietal lobes $(25.4 \%)$. In $12.2 \%$ of cases, more than three brain regions were affected simultaneously (Table S6 on 
Supplementary Material). Pathological changes in the brain, as identified by CT imaging, were found in $73 \%$ of cases, and were more frequent in males than in females (54\% vs. $17 \%, p=0.006$ ) (Table S2 on Supplementary Material). In males, the involvement of the temporal lobes was often bilateral $(p=0.005)$; in females, it was mostly unilateral (Table S7 on Supplementary Material). CT scans positive for fat emboli revealed a main peak in the age category 19 to 30 years (33\% of cases) and a second minor peak (9.5\% of cases) in those aged over 70 years of age. By contrast, the pathological findings as revealed by NMR seem to show a trimodal distribution as opposed to the two main peaks disclosed by CT scans: $32 \%$ of cases in the $19-30$ years age group; $13 \%$ of cases in the 51-60 age group; and $11 \%$ of cases aged over 70 years (Table S8 on Supplementary Material). All brain regions were affected the most in the 19-30 age category, whereas the occipital lobes were least affected in all ages (Table S9 on Supplementary Material). With regard to PFO, approx. $12 \%$ of all cases were positive (8.5\% men and $5.6 \%$ women, p. 0.47 ) - a lower incidence than reported for the whole population (25\%) [24]. In particular, PFO was present in 6\% of cases in the 19-30 years age group and $9 \%$ of cases over 70 years of age. Univariate logistic regression revealed both the male gender and the presence of rib fractures to constitute significant risk factors for FES (RR 1.874, 95\% CI 1.2722.477, $p<0.001$; and RR 0.202, 95\% CI 0.015-0.388, $p=$ 0.034 , respectively). On the other hand, multivariate logistic regression analysis revealed the male gender to be significantly associated (RR 1.412, 95\% CI 0.484-2.341, $p<0.001$ ), but not the presence of rib fractures (Table S10 on Supplementary Material).

Univariate logistic regression to assess risk factors for the development of FES in females compared with males showed age to be a correlated factor (RR 1.054, 95\% CI $1.036-1.00, p<0.001$ ) (Table S11 on Supplementary Material). Finally, it should be noted that the number of case reports published on FES has grown considerably over the last decade (2008-2018) (Figure S3, supplemetary material).

\section{Discussion}

The first case of fat embolism was described in 1863 by Bergmann [25]. The report regarded a patient who fell from a roof and sustained a comminuted fracture of the distal femur and developed dyspnea, cyanosis, and then fell into a coma $60 \mathrm{~h}$ later [25]. The patient's autopsy revealed a massive fat embolism. The syndrome resulting from a CFE had been described just the year previous by the pathologist Friedrich Albert von Zenker [26].

In the present study, by means of PRISMA analysis, we identified 170 case report studies on CFE published in the medical literature over the last 58 years (Figure
S3). Data related to 260 individual cases were extracted from these papers and subjected to meticulous statistical analysis. Table 1 on Supplementary Material summarizes the general clinical features associated with fat embolism. The data were tested for correlations between FE/ FES and fracture laterality and type, the presence of PFO and the CFE physiopathology. The majority of patients affected by FE/FES were found to be young males negative for a PFO. Although the clinical significance and relevance of the results are not always very clear, especially in relation to the laterality of the fracture, a leftsided fracture was more frequent in men compared with women, although fractures were more frequent in both sexes on the right side compared with left sided or bilateral fractures.

A femoral fracture was present in $71 \%$ of cases. We do not know whether these statistics are linked to the fact that men are more likely to be involved in road traffic accidents than women.

Our data reveal the incidence of FE/FES to be higher in bilateral femoral fractures and following intramedullary nail fixation, i.e. in the long bones of the lower limbs. Multivariate analysis revealed men to be affected by FES syndrome more frequently than women. We also reveal evidence for the percentage of patients positive for FES to drop significantly after 30 years of age, although a trend for a slight rise in the number of patients was observed in those aged 51-60 years (associated with the use of MR imaging) and over 70 years. The strong reduction in the frequency of FES after 30 years of age may signify that the energy of the impacts causing bone fractures decreases as the age of the subject increases. This supports previous reports suggesting that FES is more frequently associated with complicated trauma [27-30].

Indeed, the main finding of the analysis was a correlation between gender (male), age (being younger), NMRI findings and FES presentation. On the contrary, this correlation could explain why the degree of fat embolism is sometimes not enough to trigger FES or CFE.

Although a correlation was identified between a leftsided fracture and being male, it is unclear whether a causal relationship exists or whether it is just a statistical verdict.

Acute respiratory failure after trauma may arise due to a number of causes; for example, pneumothorax, hemothorax, airway aspiration, lung contusion or acute pulmonary embolism $[30,31]$. In this last scenario, a fat embolism can cause direct obstruction of the main branches of the pulmonary capillaries with acute right heart failure (Peltier's theory) [32]. Some papers advocate the use of thrombus-pulmonary-embolectomy; however, this procedure requires specialized cardiac centers and the potential use of extracorporeal life support 
[33-35]. Moreover, this latter treatment, due to the necessity of heparinization constitutes a relative contraindication [33].

Non-fulminant acute pulmonary embolism is the most frequent consequence of fat embolism (FE) and it shows a different degree of respiratory failure, usually with low gas exchange, secondary to ventilation-perfusion mismatch [30].

The "late" symptoms of FES, manifesting as adult respiratory distress syndrome (ARDS), occur 2-3 days after the bone trauma $(48-72 \mathrm{~h})$ and are thought to be triggered by biochemical cascades following alveolar wall damage, resulting in the liberation of free fatty acids from the lysis of triglycerides [36]. The only treatment for ARDS is patient support with non-invasive or invasive artificial mechanical ventilation, depending on the gravity. In the case of invasive mechanical ventilation, a lung protective strategy has been shown to improve the outcome in these patients [37].

In the unfortunate event of death, macroscopic examinations of the lungs were found to reveal nonspecific areas of pulmonary infarction and hemorrhagic alveolitis, whereas microscopic examinations using Oil Red O staining revealed extensive intravascular FE in the peribronchiolar and alveolar vessels with small lipid vesicles located within alveolar macrophages (Fig. 2).

Interest in a potential association between $\mathrm{PFO}$ and CFE as a putative mechanism of FES has been noted in the literature $[38,39]$. Briefly, a PFO is the persistence into adulthood of an embryonic defect in the atrial septum in the form of a right to left cardiac shunt, and it has been proposed to form a possible anatomical link between venous thrombosis and acute (or sudden) stroke [24]. The data from the present study do not provide any evidence to support this. The prevalence of PFO at autopsy in the general population is approximately $27.3 \%$ [24]. Here, we found an incidence of just $12 \%$ - less than half that of the general population. If we were able to divide the cases according to the neurological consequences of FE/FES into 'acute' and 'late' cases, the presence of PFO might be able to explain the symptomatology of the former, whereas we might find PFO absent in the latter. The late manifestation may instead be related to pulmonary complications explained by a mechanism in which free fatty acids produce local biochemical mediators of oxidative stress that damage the capillary beds as a consequence of nitric oxide (NO), free radicals and inflammatory cytokines $[36,13]$. In this event, microscopic examination of the brain would be expected to reveal perivascular cerebral oedema with areas of parenchymal and neuronal ischemic injury. Indeed, Oil Red O stained slides showed multiple intravascular fat emboli with focal areas of massive fat extravasation in patients with 'late' FES (Fig. 3), whereas brain CT scans of patients with 'acute' FES were reported to be normal. In one case, a massive cerebral oedema after acute FE was identified (Fig. 4).

With regard to central nervous system lesions, we found the prevalence CFE to be slightly higher in the basal ganglia, followed by the juxtacortical regions of the frontal, parietal and temporal lobes. It seems to reflect the different anatomical distribution of the vascular
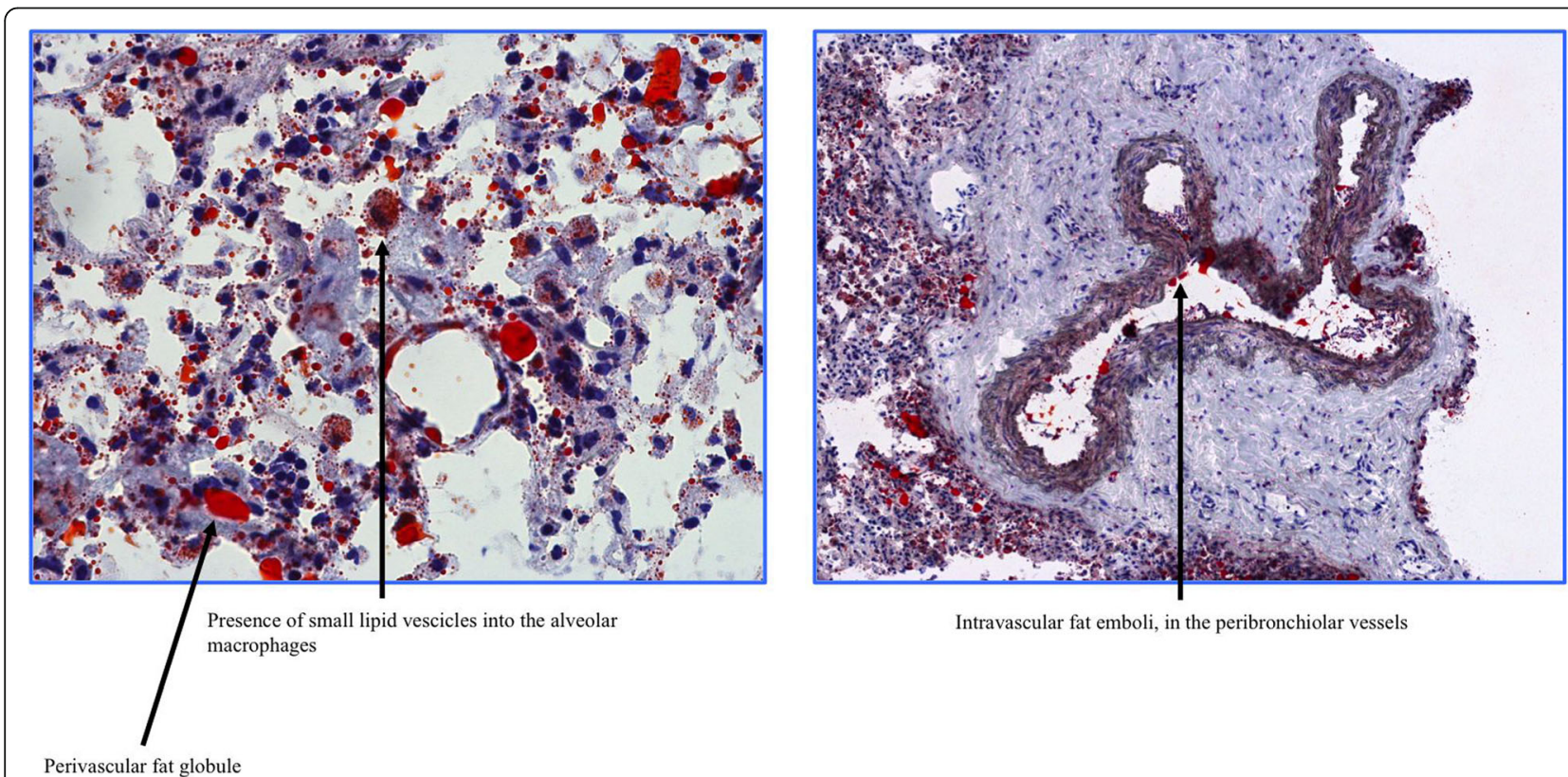

Intravascular fat emboli, in the peribronchiolar vessels

Fig. 2 Oil Red O stained slides of the lung reveal extensive intravascular fat emboli within the peribronchiolar and alveolar vessels, as well as a perivascular fat globule and the presence of small lipid vesicles within alveolar macrophages 


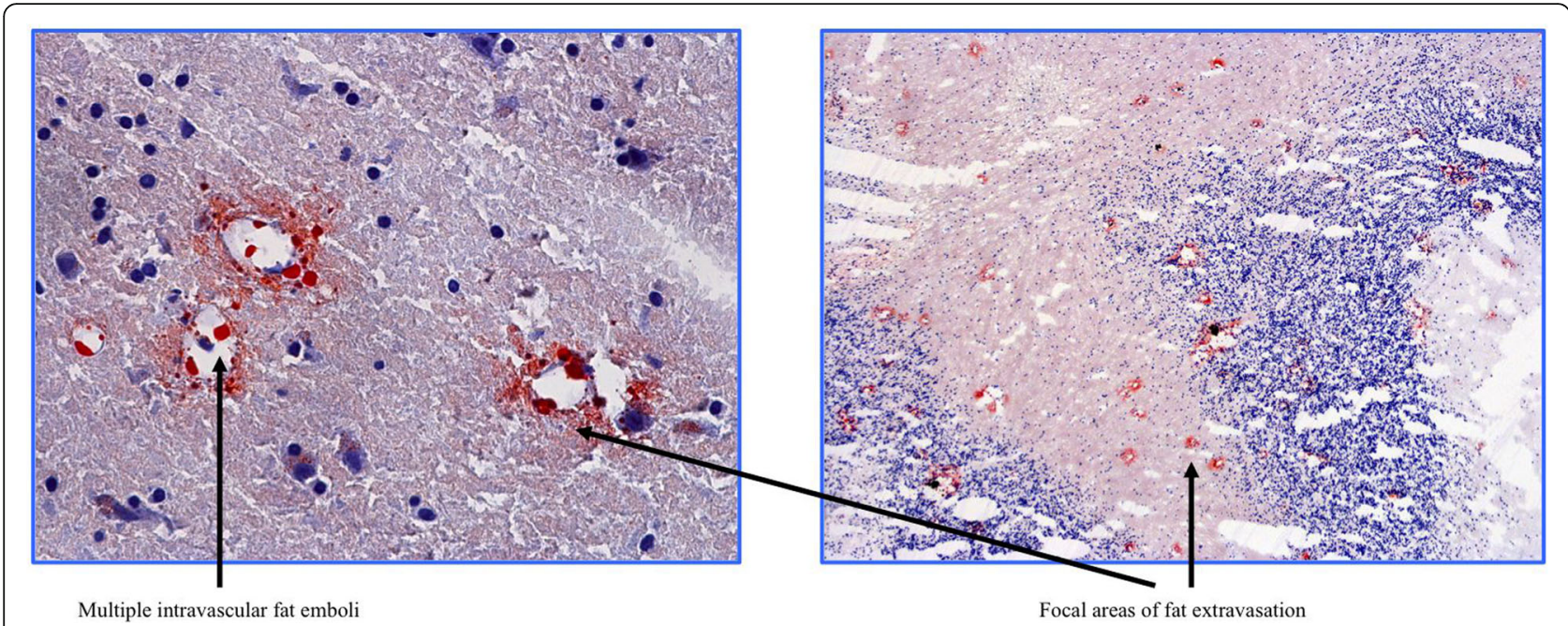

Fig. 3 Multiple intravascular fat emboli and focal areas of fat extravasation in the central nervous system of a young patient whose cause of death was a massive cerebral fat embolism

territories of the brain. In particular, there was a clear involvement of the so-called anterior circulation respect to the posterior circulation that includes the vertebrobasilar system supply, all the posterior fossa structures as well the midbrain, posterior thalami and the occipital lobes. No involvement of the posterior fossa structures was noted. It is interesting to highlight that, as in cardioembolic disease (of distinct origin), the brain's involvement is more frequently bilateral, although involvement of the right side seems to be slightly more prevalent than the left in some cases. In general, intensive care support has markedly improved the outcome of patients with FES, decreasing the incidence of fatal cases from 15 to 20 to $7 \%$ [39].

In an elegant study, Byrick et al. presented their theory of the pathophysiology of FE in which they investigated the effects of FE in 21 anesthetized mongrel dogs without PFO following cemented arthroplasty [17]. They found that the deformability of the fatty material enabled it to pass through the lung filter under the appropriate pressure, forcing the liquefied fat into the open veins (this can explain why marrow fat needs to be forced into

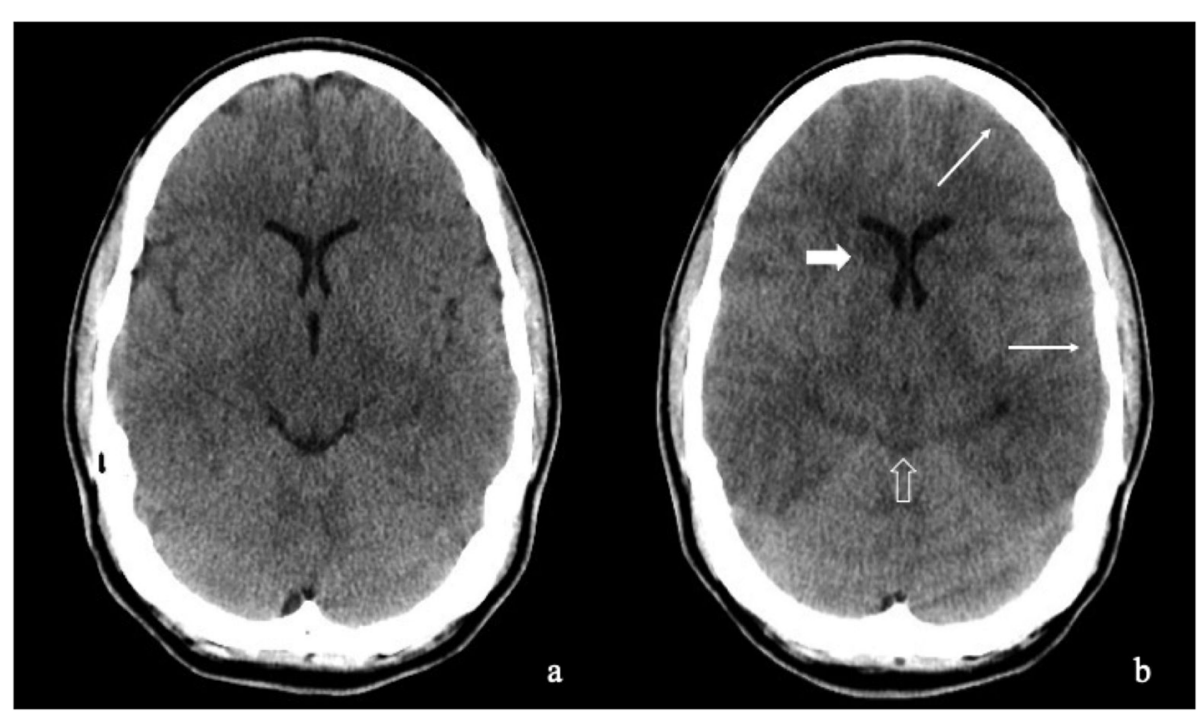

Fig. 4 An axial non enhanced computed tomography (NECT) scan performed on the first day (a) and $24 \mathrm{~h}$ later (b) show the appearance of diffuse cerebral edema with initial loss of normal gray-white matter differentiation (narrow white arrows), obliteration of CSF spaces (unfilled think arrow) and the "disappearing" of the basal ganglia (i.e., head of the right caudate nucleus) (filled thick arrow) 
the veins to reach the lungs and then the head). We propose thinking about FES using the metaphor of a 'test your strength' fairground attraction hammer game, in which FES is an 'upper-body syndrome' related to high-velocity trauma. Moreover, since the fat globule is deformable and fluid, the pulmonary vascular occlusion constitutes a transient and variable phenomenon that may arise over a period, as shown in the Byrick canine study.

When a diagnosis of FES is suspected, a cerebral NMR must be performed, followed by supportive treatments focused on the clinical symptoms, and hemodynamic and respiratory support [40].

Use of transcranial Doppler has also been suggested for the documentation of CFE [41, 42]. Figure S4 (supplementary material) shows a transcranial Doppler performed in a 19-year-old old boy that detected a microembolic signal in the left MCA following a saline/ air injection. Near infrared spectroscopy (NIRS) or Bispectral Index (BIS) might also be available in an ICU and aid monitoring patient evolution. However, the most sensitive available exam remains MR imaging, which directly provides an anatomical correlate of the clinical severity of the brain injury (Fig. 5) [40]. In some case studies, MR imaging was able to show the disappearance of brain lesions after 2 months, associated with good clinical outcome [41-45].

At the time of this review, no established guidelines exist for the treatment of FE or FES and therapy remains largely supportive. One meta-analysis has suggested that corticosteroids might be beneficial in preventing FES and hypoxia without increasing the risk of infection, although mortality was unchanged [46].

The potential therapeutic role of heparin in FE remains unknown and its use in trauma patients is neither indicated nor contraindicated. In an ideal scenario, a diagnostic methodology or blood test specific for FES would be available. Of course, other pathologies are able to cause "non-traumatic" fat embolism (NTFE), such as sickle cell crisis, fatty liver disease, total parenteral nutrition, prolonged steroid use and non-traumatic joint arthroplasty $[46,47]$. FE has been reported in the perinatal period around childbirth, although extremely rare [48].

In the last 10 years, the number of FES related published case reports has increased substantially [15, 16, 19-21]. The frequency of the disease has not undergone any significant changes, although it probably remains underdiagnosed in clinical practice.

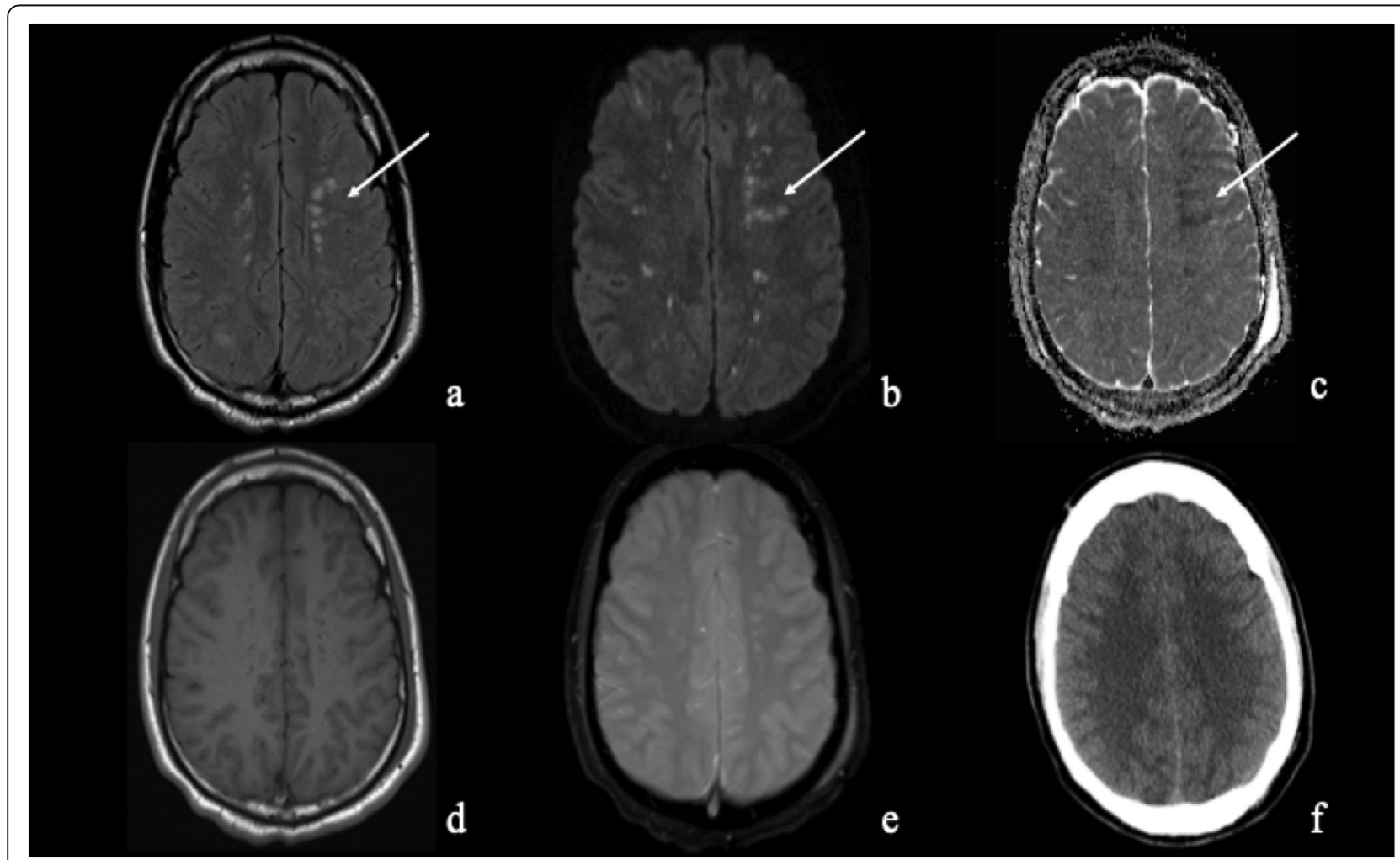

Fig. 5 Axial FLAIR (a) magnetic resonance imaging sequence shows the presence of numerous multifocal hyperintensities involving the deep white matter of both hemispheres (arrow), which correspond to innumerable tiny foci of diffusion restrictions on DWI (b) with low signal on ADC map (c) (arrow). These findings are the so-called "starfield" pattern characteristic of cerebral fat embolism syndrome. In the same patient, the SET1W (d) and T2* GRE (f) sequences, and the axial non enhanced computed tomography NECT scan (f) appear relatively normal 
The results of this structured literature analysis permitted us able to generate some hypotheses about fracture laterality, the presence of PFO and the underlying pathophysiological processes in FES that might help further our understanding of this pathology. Some limitations of the study also need to be acknowledged. Although we tried to include all case reports of adults with cerebral fat embolism published in the latest 58 years, in some of these reports, data were incomplete and as such not included in the study. Consequently, our results are based on the interpretation of the data analysis available from the detected cases.

\section{Conclusion}

From this review of clinical cases published in the past 58 years, we can conclude that FE/FES is more frequent in men aged less than 30 years following multiple fractures of the legs. It appears that the laterality of the fracture is not related to the development of the syndrome. However, FES may be more frequent after a burst fracture in which the fat marrow is pushed to the upper region of the body through the lungs. Although most cases of FES occur $48-72 \mathrm{~h}$ after a fracture, further work is required to establish whether acute brain presentation (i.e., within $24 \mathrm{~h}$ ) is related to the presence of PFO. Evidence of this could aid further research into obtaining an early diagnosis and guide a proactive approach to saving the brain from FE-induced lesions.

\section{Supplementary Information}

The online version contains supplementary material available at https://doi. org/10.1186/s13049-021-00861-x.

Additional file 1: Figure S1. Study flow diagram according to the PRIS MA reporting system.

Additional file 2: Figure S2. Bar chart showing the distribution of case reports according to patient gender and age.

Additional file 3: Figure S3. Case reports included in this review for each year since 1960.

Additional file 4: Figure S4. The figure above shows 3 MES detected in the left MCA after saline/air injection in a 19-year-old boy admitted to the Emergency Department following a car crash. At the medical examination, he was awake, cooperative and interacted regularly. Radiological findings demonstrated a femur fracture, a radius and ulna fracture, and a hip fracture, all of which were left-sided. After $36 \mathrm{~h}$ of hospital admission, the patient underwent surgical repair of the femur and upper arm fractures. Given increasing drowsiness and hypoxemia after the operation, the patient was admitted to the ICU. A neuro diffusion-weighted MRI showed the classic "starfield" appearance of multiple foci of restricted diffusion with circled areas - pathognomonic evidence of cerebral fat embolism. The patient did not require endotracheal intubation or mechanical ventilation. After $72 \mathrm{~h}$, he was transferred to the Neurology Department, where his clinical condition improved to the point of being discharged.

Additional file 5: Table S1. Key characteristics of case reports. Table S2. Main differences between men and women. Table S3. Fracture site. Table S4. Fracture laterality in men vs. women. Table S5. Type and laterality of fracture per age category. Table S6. Characteristics of brain lesions identified by neuroimaging. Table S7. Location of central nervous system involvement according to gender. Table S8. Percentage of cases per age category positive for PFO and fat emboli as ascertained by brain imaging. Table S9. Anatomic distribution of cerebral lesions per age category. Table S10. Univariate and multivariate logistic regression for assessing risk factors with increasing age. Table S11. Univariate and multivariate logistic regression for assessing risk factors in woman vs. men with fat cerebral embolism.

\section{Acknowledgements}

None.

\section{Authors' contributions}

LV designed and supervised the review; EB supervised the review; CD designed the review, was involved in literature research; FB supervised; MV performed statistical analysis; $\mathrm{MO}$ took care and revised the pathological mechanisms; DB took care of the radiological part; CI, FM, FS, and MS were involved with literature research; DO drafted the manuscript, the edited version and the supervised the review; MR and TB supervised the review. The author(s) read and approved the final manuscript.

\section{Funding}

No fund was used for this study.

Availability of data and materials

Data are available following a reasoned request.

\section{Declarations}

Ethics approval and consent to participate N/A.

Consent for publication

N/A.

Competing interests

Prof. Vetrugno received travel support from Cook Medical Inc. No other conflicts of interest have to be declared for the remaining authors.

\section{Author details}

${ }^{1}$ Department of Anesthesia and Intensive Care, Anesthesia and Intensive Care Clinic, Azienda Sanitaria Universitaria Friuli Centrale, Udine, Italy. ${ }^{2}$ Department of Medicine, Anesthesia and Intensive Care Clinic, University of Udine, Udine, Italy. ${ }^{3}$ Department of Medicine and Surgery, Unit of Anesthesiology, Parma University Hospital, Parma, Italy. ${ }^{4}$ Department of Anesthesia and Intensive Care, Anesthesia and Intensive Care Unit 1, Azienda Sanitaria Universitaria Friuli Centrale, Udine, Italy. ${ }^{5}$ Department of Anesthesia and Intensive Care, Anesthesia and Intensive Care Unit 2, Azienda Sanitaria Universitaria Friuli Centrale, Udine, Italy. ${ }^{6}$ Department of Neurosciences, Reproductive and Odontostomatological Sciences, University of Naples "Federico II", Naples, Italy. ${ }^{7}$ Department of Medicine, Surgical Pathology Section, University of Udine, Udine, Italy. ${ }^{8}$ Department of Diagnostic Imaging, Neuroradiology Unit, Azienda Sanitaria Universitaria Friuli Centrale, Udine, Italy. ${ }^{9}$ School of Medicine and Surgery, University of Milan-Bicocca, Monza, Italy.

${ }^{10}$ Department of Medicine, Maxillofacial Surgery, University of Udine, Udine, Italy. ${ }^{11}$ Azienda Sanitaria Universitaria Friuli Centrale, Maxillofacial Surgery, Udine, Italy.

Received: 1 June 2020 Accepted: 4 March 2021

Published online: 12 March 2021

References

1. Bulger EM, Smith DG, Maier RV, et al. Fat embolism syndrome. A 10-year review. Arch Surg. 1997;132:439.

2. Fabian TC, Hoots AV, Stanford DS, et al. Fat embolism syndrome: prospective evaluation in 92 fracture patients. Crit Care Med. 1990;18(42):46.

3. Aman J, van Koppenhagen L, Snoek AM, et al. Cerebral fat embolism after bone fractures. Lancet. 2015;386:e16.

4. Gauss H. The pathology of fat embolism. Arch Surg. 1924;9(592):605.

5. Lehman EP, Moore RM. Fat embolism, including experimental production without trauma. Arch Surg. 1927;14(621):662. 
6. Pell AC, Hughes D, Keating J, et al. Brief report: fulminating fat embolism syndrome caused by paradoxical embolism through a patent foramen ovale. N Engl J Med. 1993;329:926-9.

7. Fabian TC. Unraveling the fat embolism syndrome. NEJM. 1993;329:961-3.

8. Gurd AR. Fat embolism: an aid to diagnosis. J Bone Joint Surg (Br). 1970;52: 732-7.

9. Gurd AR, Wilson RI. The fat embolism syndrome. J Bone Joint Surg (Br). 1974;56-B:408-16.

10. Peltier LF. Fat embolism: a perspective. Clin Orthop. 1988;232:263-70.

11. Kellogg RG, Fontes RBV, Lopes DK. Massive cerebral involvement in fat embolism syndrome and intracranial pressure management. J Neurosurg. 2013;119:1263-70

12. Lee $\mathrm{SC}$, Yoon JY, Nam CH, et al. Cerebral fat embolism syndrome after simultaneous bilateral total knee arthroplasty a case series. J Arthroplast. 2012;27:409-14

13. Godoy DA, Di Napoli M, Rabinstein AA. Cerebral fat embolism: recognition, complications, and prognosis. Neurocrit Care. 2018;29:358-65.

14. Whitenack SH, Hausberger FX. Intravasation of fat from the bone marrow cavity. Am J Pathol. 1971:65:335-46.

15. Etchells EE, Wong DT, Davidson G, et al. Fatal cerebral fat embolism associated with a patent foramen ovale. Chest. 1993;104:962-3.

16. Mijalski C, Lovett A, Mahajan R, et al. Cerebral fat embolism: a case of rapidonset coma. Stroke. 2015;46:e251-3.

17. Byrick RJ, Mullen JB, Mazer CD, et al. Transpulmonary systemic fat embolism Studies in mongrel dogs after cemented arthroplasty. Am J Respir Crit Care Med. 1994;150:1416-22.

18. Jorgensen A, Bashir A, Satpathy J. Cerebral fat embolism syndrome (FES): similar cases with different outcomes. BMJ Case Rep. 2018;2018: bcr2018225261.

19. Shacklock E, Gemmell A, Hollister N. Neurological effects of fat embolism syndrome: a case report. J Intensive Care Soc. 2017:18:339-41.

20. Huber-Lang M, Brinkmann A, Straeter J, et al. An unusual case of early fulminant post-traumatic fat embolism syndrome. Anaesthesia. 2005;60: $1141-3$.

21. Caricato A, Russo G, Biasucci DG, et al. Fat embolism syndrome. Intensive Care Med. 2017:43:1411-2.

22. Goenka N, Roppe AN. 13 images in clinical medicine. Cerebral fat embolism. N Engl J Med. 2012;367:1045

23. Moher $D$, Shamseer $L$, Clarke $M$, et al. Preferred reporting items for systematic review and meta-analysis protocols (PRISMA-P) 2015 statement. Syst Rev. 2015;4:1.

24. Pauline Y, Andrew KY, Subramaniam B, et al. Association of preoperatively diagnosed patent foramen ovale with perioperative ischemic stroke. JAMA. 2018:319:452-62.

25. Von Bergmann E. Ein fall todlicher fettenbolic. Berl Klin Wochenscher. 1873; 10:385.

26. Zencker FA. Beitrage zur normalen und pathologischen anatomic der lunge, vol. 31. Dresden: J Braunsdorf; 1862

27. Kosova E. Fat embolism syndrome. Circulation. 2015;131:317-20.

28. Randelli F, Capitani P, Pace F, Favilla S, Galante C, Randelli P. Bilateral femoral shaft fractures complicated by fat and pulmonary embolism: a case report. Injury. 2015;46:S28-30

29. Singh S, Singh SU, Dwivedi D, Kaur KB. Early recognition and successful management of a case of fracture shaft of the femur complicated with fat embolism syndrome and acute respiratory distress syndrome. J Mar Med Soc. 2020;22:94-5.

30. Aggarwal R, Banerjee A, dev Soni K, Kumar A, Trikha A. Clinical characteristics and management of patients with fat embolism syndrome in level I apex trauma centre. Chin J Traumatol. 2019;22:172-6.

31. Watkins TR, Nathens AB, Cooke CR, Psaty BM, Maier RV, Cuschieri J, Rubenfeld GD. Acute respiratory distress syndrome after trauma: development and validation of a predictive model. Crit Care Med. 2012;40 2295-303. https://doi.org/10.1097/CCM.0b013e3182544f6a.

32. George J, George R, Dixit R, Gupta RC, Gupta N. Fat embolism syndrome. Lung India. 2013:30:47-53.

33. Jorens PG, Van Marck E, Snoeckx A, Parizel PM. Nonthrombotic pulmonary embolism. Eur Respir J. 2009:34:452-74.

34. Ramesh D, Setty HS, Kumarswamy, Kumar S, Jayanth, Manjunath CN. Timely embolectomy in acute massive pulmonary embolism prevents catastrophe: an experience from two cases. J Nat Sci Biol Med. 2016;7:176-9.
35. Marshall PS, Mathews KS, Siegel MD. Diagnosis and management of lifethreatening pulmonary embolism. J Intensive Care Med. 2011:26:275-94.

36. Karagiorga G, Nakos G, Galiatsou E, Lekka ME. Biochemical parameters of bronchoalveolar lavage fluid in fat embolism. Intensive Care Med. 2006; 32(1):116-23.

37. Griffiths MJD, McAuly DF, Perkins GD, Barrett N, Blackwood B, Boyle A, Chee N, Connolly B, Dark P, Finney S, Salam A, Silversides J, Tarmey N, Wise MP, Baudouin SV. Guidelines on the management of acute respiratory distress syndrome. BMJ Open Respir Res. 2019;6:e000420. https://doi.org/10.1136/ bmiresp-2019-000420.

38. Nikolić S, Zivković V, Babić D, Djonić D, Djurić M. Systemic fat embolism and the patent foramen ovale--a prospective autopsy study. Injury. 2012;43(5): $608-12$.

39. Newbigin K, Souza CA, Torres C, Marchiori E, Gupta A, Inacio J, Armstrong $M$, Peña E. Fat embolism syndrome: state-of-the-art review focused on pulmonary imaging findings. Respir Med. 2016;113:93-100.

40. Kuo KH, Pan YJ, Lai YL, Cheung WK, Chang FC, Jarosz J. Dynamic MR imaging patterns of cerebral fat embolism: a systematic review with illustrative cases. Am J Neuroradiol. 2014;35:1052-7.

41. Forteza AM, Koch S, Romano JG, et al. Transcranial Doppler detection of fat emboli. Stroke. 1999;30:2687-91.

42. Forteza AM, Koch S, Campo-Bustillo I, Gutierrez J, Haussen DC, Rabinstein AA, Romano J, Zych GA. Transcranial doppler detection of cerebral fat emboli and relation to paradoxical embolism: a pilot study. Circulation. 2011;10(123):1947-52.

43. Srikanth $\mathrm{K}$, Sundararajan $\mathrm{S}$, Rajasekaran $\mathrm{S}$. Late recovery in cerebral fat embolism. Indian J Orthop. 2014;48:100-3.

44. Stoeger A, Daniaux M, Felber S, Stockhammer G, Aichner F, zur Nedden D. MRI findings in cerebral fat embolism. Eur Radiol. 1998;8:1590-3.

45. Herway ST, Slotto J, Harlan E, Newhouse B. Cerebral fat embolismsyndrome. Anesthesiology. 2016;124:1167.

46. Bederman SS, Bhandari M, McKee MD, Schemitsch EH. Do corticosteroids reduce the risk of fat embolism syndrome in patients with long-bone fractures? A meta-analysis. Can J Surg. 2009;52(5):386-93.

47. Sakashita M, Sakashita S, Sakata A, Uesugi N, Hyodo I, Noguchi M. An autopsy case of non-traumatic fate embolism syndrome. Pathol Int. 2017;67: $477-82$.

48. Schrufer-Poland T, Singh P, Jodicke C, Reynolds S, Maulik D. Nontraumatic fat embolism found following maternal death after cesarean delivery. AJP Rep. 2015;5:e1-5. https://doi.org/10.1055/s-0034-1394152.

\section{Publisher's Note}

Springer Nature remains neutral with regard to jurisdictional claims in published maps and institutional affiliations.

Ready to submit your research? Choose BMC and benefit from

- fast, convenient online submission

- thorough peer review by experienced researchers in your field

- rapid publication on acceptance

- support for research data, including large and complex data types

- gold Open Access which fosters wider collaboration and increased citations

- maximum visibility for your research: over $100 \mathrm{M}$ website views per year

At BMC, research is always in progress.

Learn more biomedcentral.com/submissions 